\title{
Practical stabilisation of switched affine systems with dwell-time guarantees
}

\author{
C. Albea Sanchez, G. Garcia, S. Hadjeras, W.P.M.H. Heemels, L. Zaccarian
}

\begin{abstract}
Using a hybrid systems approach, we address the practical stabilization of operating points for switched affine systems, ensuring a minimum dwell time and an admissible chattering around the operating point. Two different solutions are shown to induce uniform dwell time, based on time- or space-regularization. The proposed solutions provide useful tuning knobs to separately adjust the switching frequency during transients and at the steady state. The strengths of the method are illustrated by simulating a boost converter.
\end{abstract}

\section{INTRODUCTION}

Switched systems are key to modeling several physical control systems. Among them, switched affine systems (SAS) are given by

$$
\begin{aligned}
\dot{x} & =A_{\sigma} x+a_{\sigma}, \quad x(0)=x_{0} \\
z & =C x,
\end{aligned}
$$

where the control input $\sigma: \mathbb{R}_{\geq 0} \rightarrow \mathcal{N}:=\{1,2, \ldots, N\}$ is the switching signal, assigning a specific desired mode among $N$ possible ones at each time. Moreover, in dynamics (1), $x \in \mathbb{R}^{n}$ is the state, $z \in \mathbb{R}^{p}$ is a performance output, and $A_{i}$ and $a_{i}$ have suitable dimensions for all $i \in \mathcal{N}$.

Many works have been published in the past twenty years about stabilization of Switched Linear Systems (SLS), being SAS with $a_{i}=0, i \in \mathcal{N}$ (see, e.g., [5], [6], [12], [23] and references therein). For SLS, the objective is to stabilize the origin, which is a common equilibrium to all the dynamics. Indeed, homogeneity of the SLS dynamics is exploited in those works, thereby simplifying the control design providing, among other things, direct tools to show uniform upper bounds on the switching rate (dwell time) of the input along the ensuing solutions. The goal of stabilizing an operating point $x_{e} \in \mathbb{R}^{n}$, which is the more general objective in the SAS case, is much more challenging. Indeed, in the general case considered in this paper $x_{e}$ is not a common equilibrium with the consequence that asymptotic stabilization of $x_{e}$ is not possible with finite switching rates (uniform dwell time). Indeed, the fact that $x_{e}$ is not a common equilibrium implies that arbitrarily fast switching is needed by any

C. Albea Sanchez, G. Garcia and S. Hadjeras are with CNRS, LAAS, Université de Toulouse, 7 avenue du colonel Roche, F-31400 Toulouse, France shadjeras, calbea, garcia@laas.fr

W.P.M.H. Heemels is with the Control Systems Technology group, Department of Mechanical Engineering, Eindhoven University of Technology, The Netherlands m.heemels@tue.nl

L. Zaccarian is with CNRS, LAAS, Université de Toulouse, 7 avenue du colonel Roche, F-31400 Toulouse, France and Dipartimento di Ingegneria Industriale, University of Trento, Italy zaccarian@laas.fr asymptotic stabilizer as the solutions approach $x_{e}$. This substantial difference calls upon alternative solutions and proof techniques not relying on the homogeneity properties enjoyed by the SLS case.

Important results towards stabilization of operating points in SAS have appeared in [3], [9], [17], [20], and also for switched systems with a general nonlinear form [14], [15]. However, these results can induce sliding modes and generate arbitrarily fast switching already during the transients (in addition to the above-mentioned steadystate issues). Alternative approaches, inducing a positive dwell time, can be found in the context of specific SAS applications, such as power converters [2], [18], [22]. In these works the authors aim at ensuring a dwell time associated with an admissible chattering around the operating point. Nevertheless, [2] does not prove a minimum time associated to space regularization, [22] is focused on a specific power converter application (boost converter) and [18] does not provide a full stability proof. Interesting results are also reported in [21], which ensures dwell time properties of a class of systems of the type (1), where $a_{\sigma}$ is a bounded function of time, possibly converging to zero. However, only a mild boudedness property is proven in [21] for the case of non-vanishing converging $a_{\sigma}$, which is the setting that we consider here. The results in [8] and [10], address the general class (1) and are based on the use of sampled-data controllers guaranteeing practical stability for a periodic switching controller. Nonlinear switched systems are addressed in [24], which focuses on discrete-time systems by using a dwell-time $H_{\infty}$ control. Finally, both space and time- regularization are proposed without using Lyapunov functions in [4], which has limited applicability because the discontinuities may occur only in a set given by a smooth manifold of dimension 1 for the time-regularization case and in a discontinuity surface having co-dimension 2 for the space-regularization case.

Summarizing the above, control of SAS can exhibit transient chattering (or lack of dwell time guarantees) possibly due to the presence of sliding modes in the proposed controller and then are bound to unavoidable steady-state chattering when approaching the operating point. While the first problem can be avoided by a suitable control action, the second one requires resorting to practical (rather than asymptotic) stability guarantees. Motivated by this fact, in this paper, we address practical stabilization of an operating point $x_{e}$ for SAS (1), ensuring the following features:

1) stabilization of an (arbitrarily small) set around the operating point $x_{e}$ whose size can be adjusted by design parameters; 
2) a positive minimal dwell time between consecutive switches during the transient and steady-state response, which can be adjusted by the design parameters, to warrant practical implementability,

3) trade-off knobs (design parameters) that can be suitably adjusted to favor dwell-time properties versus performance guarantees (an LQ cost for the transient phase and the size of the stabilized set for the steadystate phase).

Following the notation of the above cited works [2], [18], [22], we guarantee a positive dwell time by modifying (regularizing) the chattering controllers using either space or time regularization techniques, where the former enforces dwell time using space-based hystheresis logics, and the latter uses instead an explicit timer inhibiting switches up to some guaranteed dwell time. By casting the practical stabilization problem using the recent hybrid framework of [7], we show that time and space regularizations are two variations of a central result, consisting in our Lemma 2 in Section $\mathrm{V}$, which provides an elegant and unified view of the two approaches. To the best of our knowledge, such a unified view, and the distinction between transient and steady-state chattering avoidance has not been proposed before, but only scattered results, perhaps for specific subclasses of (1) such as SLS or specific applications, are available in the literature. Rigorous proofs are given for our global and practical stability statements, using the hybrid Lyapunov theory in [7] and its extensions in [16], [19].

The paper is organized as follows. Section II provides the problem formulation, while Section III describes the proposed solution while Section IV discusses optimalitybased parameter tuning. Time- and space-regularizations inducing dwell time and practical stability are introduced and characterized in Section V. Finally, Section VI discusses an illustrative example and Section VII draws concluding remarks.

\section{Problem SETUP}

We aim to provide feedback strategies determining $\sigma$ such that practical stabilisation of an operating point $x_{e} \in$ $\mathbb{R}^{n}$ for SAS (1) is achieved while satisfying requirements $1), 2$ ) and 3 ) of the introduction. To this end, we make the following standard assumption (see [3], [10], [13], [21]).

Assumption 1: Given $\Lambda:=\left\{\lambda \in[0,1]^{N} \mid \sum_{i=1}^{N} \lambda_{i}=1\right\}$, there exists $\lambda \in \Lambda$, such that

$$
\sum_{i=1}^{N} \lambda_{i}\left(A_{i} x_{e}+a_{i}\right)=0, \text { and } \sum_{i=1}^{N} \lambda_{i} A_{i} \text { is Hurwitz. }
$$

Definition 1: The set of admissible operating points $\Omega_{e} \subset \mathbb{R}^{n}$ is given by

$$
\Omega_{e}:=\left\{x_{e} \in \mathbb{R}^{n} \mid \exists \lambda \in \Lambda \text { satisfying (2) }\right\} .
$$

Hence, $x_{e} \in \Omega_{e}$ if it is an equilibrium point for the averaged dynamics

$$
\dot{x} \in F(x):=\left\{\sum_{i=1}^{N} \lambda_{i}\left(A_{i} x+a_{i}\right) \mid \lambda \in \Lambda\right\},
$$

and a stability condition is satisfied on the corresponding convex dynamics. See also [2], [3], [10], [13] and the discussion in [1, Remark 1]. While requirement $x_{e} \in \Omega_{e}$ may appear to be non-restrictive for stabilizability of $x_{e}$ from $\sigma$, it is already known that this condition is not necessary even for the case of SLSs with $N=2, a_{1}=a_{2}=0$ and $x_{e}=0$, as commented in [12, Section 3.4.2]. The average dynamics can be perceived as the result of arbitrarily fast switching and as the solution of the differential inclusion (4). Such generalizations are well characterized in the context of hybrid inclusions of [7], by way of solutions corresponding to the so-called hybrid arcs. In this paper we adopt that framework and discuss properties of those hybrid arcs for hybrid formulations of SAS (1).

More specifically, we address the following problem: Given the $S A S$ (1), for each $x_{e} \in \Omega_{e}$ design a feedback law for the switching signal $\sigma$ that globally asymptotically stabilizes an arbitrarily small neighborhood of $x_{e}$ by suitably adjusting the design parameters (in other words, a parametric feedback that practically stabilizes $x_{e}$ ), while satisfying requirements 2) and 3) discussed in the introduction.

\section{CONTROL SOLUTION AND HYBRID MODEL}

Consider (1), $x_{e} \in \Omega_{e}$ and $\lambda_{e} \in \Lambda$ satisfying (2). We select two matrices $P$ and $Q$ as follows

Property 1: Matrices $P=P^{T}>0 \in \mathbb{R}^{n \times n}$ and $Q=$ $Q^{T}>0 \in \mathbb{R}^{n \times n}$ satisfy,

$$
\left(\sum_{i=1}^{N} \lambda_{e, i} A_{i}^{T}\right) P+P\left(\sum_{i=1}^{N} \lambda_{e, i} A_{i}\right)+2 Q \leq 0 .
$$

Clearly due to $\sum_{i=1}^{N} \lambda_{e, i} A_{i}$ being Hurwitz matrices $P, Q$ satisfying Property 1 always exist. Note that Property 1 , which can be already found in [21] and in the recent work [9], imposes less restrictive assumptions than in our preliminary work [1], which corresponds to a special case.

Following the formalism in [7], we propose a hybrid controller having state $\sigma$, giving the closed loop

$$
\mathcal{H}:\left\{\begin{array}{l}
{\left[\begin{array}{c}
\dot{x} \\
\dot{\sigma}
\end{array}\right]=f(x, \sigma), \quad(x, \sigma) \in \mathcal{C}} \\
x^{+} \\
\sigma^{+}
\end{array}\right] \in G(x, \sigma), \quad(x, \sigma) \in \mathcal{D},
$$

where $f$ is the flow map and $G$ is a (set-valued) jump map capturing the switching logic, selected as

$$
\begin{aligned}
& f(x, \sigma):=\left[\begin{array}{c}
A_{\sigma} x+a_{\sigma} \\
0
\end{array}\right] \\
& G(x, \sigma):=\left[\begin{array}{c}
x \\
\underset{i \in \mathcal{N}}{\operatorname{argmin}}\left(x-x_{e}\right)^{T} P\left(A_{i} x+a_{i}\right)
\end{array}\right]
\end{aligned}
$$

and where the so-called "flow" and "jump" sets $\mathcal{C}$ and $\mathcal{D}$ encompass, respectively, the regions in the (extended) space $(x, \sigma)$ where the switching strategy continues with the current mode $\sigma$ ( $\operatorname{set} \mathcal{C}$ ) or switches to a new mode (set $\mathcal{D})$. They are selected as

$$
\begin{aligned}
\mathcal{C} & :=\left\{(x, \sigma): \tilde{x}^{T} P\left(A_{\sigma} x+a_{\sigma}\right) \leq-\eta \tilde{x}^{T} Q \tilde{x}\right\} \\
\mathcal{D} & :=\left\{(x, \sigma): \tilde{x}^{T} P\left(A_{\sigma} x+a_{\sigma}\right) \geq-\eta \tilde{x}^{T} Q \tilde{x}\right\}
\end{aligned}
$$


where $\tilde{x}:=x-x_{e}$ and scalar $\eta \in(0,1)$ is a design parameter that will be shown to be useful for achieving a trade-off between the transient switching frequency and a quadratic integral performance level, as characterized later in Theorem 2. The next lemma is an instrumental step to prove our main stability result.

Lemma 1: Consider $x_{e} \in \Omega_{e}, \lambda_{e} \in \Lambda$ satisfying (2) and matrices $P \in \mathbb{R}^{n \times n}$ and $Q \in \mathbb{R}^{n \times n}$, satisfying Property 1 . Then, for each $x \in \mathbb{R}^{n}$, denoting $\tilde{x}:=x-x_{e}$,

$$
\min _{i \in \mathcal{N}} \tilde{x}^{T} P\left(A_{i} x+a_{i}\right) \leq-\tilde{x}^{T} Q \tilde{x}=-|\tilde{x}|_{Q}^{2} .
$$

Proof. The proof is a slight variation of the derivations in $[3$, eq. (12)] and is therefore omitted for the sake of compactness.

Following up on standard stability theory for hybrid systems [7], we establish uniform global asymptotic stability of the compact attractor

$$
\mathcal{A}:=\left\{(x, \sigma): x=x_{e}, \sigma \in \mathcal{N}\right\} .
$$

Theorem 1: Consider $x_{e} \in \Omega_{e}, \lambda_{e} \in \Lambda$ satisfying (2) and matrices $P \in \mathbb{R}^{n \times n}$ and $Q \in \mathbb{R}^{n \times n}$, satisfying Property 1 . Attractor (11) is uniformly globally asymptotically stable (UGAS) for hybrid system (6)-(9).

Proof. Let us take the candidate Lyapunov function

$$
V(\tilde{x}):=\frac{1}{2}|\tilde{x}|_{P}^{2}:=\frac{1}{2} \tilde{x}^{T} P \tilde{x} .
$$

If $(x, \sigma) \in \mathcal{C}$, using $(8)$ we get along flows

$$
\langle\nabla V(\tilde{x}), f(\tilde{x}, \sigma)\rangle=\tilde{x}^{T} P\left(A_{\sigma}\left(\tilde{x}+x_{e}\right)+a_{\sigma}\right) \leq-\eta \tilde{x}^{T} Q \tilde{x} .
$$

Across jumps, for $(x, \sigma) \in \mathcal{D}$, we get, from $x^{+}=x$,

$$
V\left(\tilde{x}^{+}\right)-V(\tilde{x})=\frac{1}{2}\left\{\tilde{x}^{T} P \tilde{x}-\tilde{x}^{T} P \tilde{x}\right\}=0 .
$$

Uniform global asymptotic stability is then shown by [16, Th 1]. In particular, since the distance of $x$ to the attractor (11) is defined by $|x|_{\mathcal{A}}=|\tilde{x}|$, we have that $[16$, eq. (6)] holds from the structure of $V$ and from (13) and (14). Following [16, Th 1], we build the hybrid restriction $\mathcal{H}_{\delta, \Delta}$ by intersecting $\mathcal{C}$ and $\mathcal{D}$ with

$$
S_{\delta, \Delta}:=\{(\tilde{x}, \sigma):|\tilde{x}| \geq \delta \quad \text { and } \quad|\tilde{x}| \leq \Delta\}
$$

and then proving (semi-global) practical persistence flow for $\mathcal{H}_{\delta, \Delta}$, for each fixed values of $(\delta, \Delta)$. In particular, practical persistent flow amounts to showing that there exists $\gamma \in \mathcal{K}_{\infty}$ and $M \geq 0$, such that, all solutions to $\mathcal{H}_{\delta, \Delta}$ satisfy

$$
t \geq \gamma(j)-M, \quad \forall(t, j) \in \operatorname{dom} \xi
$$

where $\operatorname{dom} \xi=\bigcup_{j \in \operatorname{dom}_{j} \xi}\left[t_{j}, t_{j+1}\right] \times\{j\}$ is the hybrid time domain (see [7, Ch. 2] for details). To establish (16), notice that after each jump, from the definition of $G$ in (7) and from (10), we have

$$
\tilde{x}^{T}\left(A_{\sigma^{+}} x+B_{\sigma^{+}}\right) \leq-\tilde{x}^{T} Q \tilde{x}<-\eta \tilde{x}^{T} Q \tilde{x},
$$

where we used $\eta<1$ and $(0, \sigma) \notin S_{\delta, \Delta}$. Therefore, if any solution to $\mathcal{H}_{\delta, \Delta}$ performs a jump from $S_{\delta, \Delta}$, it will remain in $S_{\delta, \Delta}$ (because $\tilde{x}$ remains unchanged) and then, from (9), it must jump to the interior of the flow set $\mathcal{C} \cap S_{\delta, \Delta}$. Moreover, from the strict inequality in (17), all such solutions must flow for some time after the jump and since $\mathcal{C} \cap S_{\delta, \Delta}$ is bounded, continuity of solutions with respect to initial conditions [7, Prop. 6.14] implies that there is a uniform dwell time $\rho(\delta, \Delta)$ between each pair of consecutive jumps (the well defined minimum over such flowing times over the compact set). This dwell time $\rho(\delta, \Delta)$ clearly implies $\left[16\right.$, eq. (4)] with the class $\mathcal{K}_{\infty}$ function $\gamma(j)=\rho(\delta, \Delta) j$ and $M=1$. Then, all the assumptions of [16, Theorem 1] hold and UGAS of $\mathcal{A}$ is concluded.

\section{PARAMETER TUNing FOR GUARANTEed PERFORMANCE}

While Theorem 1 addresses stability, we provide here performance guarantees for the closed loop (6)-(9), which follows the same paradigm as the one discussed, in a continuous-time setting, in [3]. This performance guarantee, may, for example, refer to desirable levels of dissipated energy, current peak, response time, among others.

Within the considered hybrid context, as discussed in the proof of Theorem 1, solutions are parametrized in a hybrid time domain. Then, we use a quadratic performance metric focusing on the flowing aspect of hybrid solutions:

$$
J(\xi):=\sum_{k \in \operatorname{dom}_{j} \xi} \int_{t_{k}}^{t_{k+1}}|\tilde{z}(\tau, k)|^{2} d \tau,
$$

where $\xi=(x, \sigma): \operatorname{dom} \xi \rightarrow \mathbb{R}^{n} \times \mathcal{N}$ is a solution to hybrid system (6)-(9), whose domain is characterized by jump times $t_{k}, k \in \mathbb{N} \geq 1$ (namely times $t_{k} \geq 0$ such that both $\left(t_{k}, k-1\right)$ and $\left(t_{k}, k\right)$ belong to $\left.\operatorname{dom} \xi\right)$, and $\tilde{z}(t, j):=$ $C \tilde{x}(t, j)$ for all $(t, j) \in \operatorname{dom} \xi$ is a suitable performance output. For these hybrid solutions, we may then give the following guarantee on the performance cost (18).

Theorem 2: Consider hybrid system (6)-(9) satisfying Assumption 1 and Property 1. If

$$
C^{T} C \leq Q
$$

then the following bound holds along any solution $\xi=$ $(x, \sigma)$ of $(6)-(9)$ :

$$
J(\xi) \leq \frac{1}{2 \eta} \tilde{x}(0,0)^{T} P \tilde{x}(0,0)=\frac{1}{2 \eta}|\tilde{x}(0,0)|_{P}^{2},
$$

where $\tilde{x}(t, j)=x(t, j)-x_{e}$, for all $(t, j) \in \operatorname{dom}(\xi)$.

Proof. Consider any solution $\xi=(x, \sigma)$ to $\mathcal{H}$. For each $(t, j) \in \operatorname{dom} \xi$, denoting $t=t_{j+1}$ to simplify notation, we have from (13)

$$
\begin{aligned}
& V(\tilde{x}(t, j))-V(\tilde{x}(0,0))=\sum_{k=0}^{j} V\left(\tilde{x}\left(t_{k+1}, k\right)\right)-V\left(\tilde{x}\left(t_{k}, k\right)\right) \\
& =\sum_{k=0}^{j} \int_{t_{k}}^{t_{k+1}}\langle\nabla V(\tilde{x}(\tau, k)), f(x(\tau, k), \sigma(\tau, k))\rangle d \tau \\
& \leq \sum_{k=0}^{j} \int_{t_{k}}^{t_{k+1}}-\eta|\tilde{x}(\tau, k)|_{Q}^{2} d \tau \leq-\eta \sum_{k=0}^{j} \int_{t_{k}}^{t_{k+1}}|\tilde{x}(\tau, k)|_{C^{T} C}^{2} d \tau,
\end{aligned}
$$


where the last inequality comes from (19). Considering $\tilde{z}(\tau, k)=C \tilde{x}(t, k)$, taking the limit as $t+j \rightarrow+\infty$ and using the fact that UGAS established in Theorem 1 implies $\lim _{t+j \rightarrow+\infty} V(\tilde{x}(t, j))=0$, we get from (21), $\eta J(\xi) \leq V(\tilde{x}(0,0))=\frac{1}{2}|\tilde{x}(0,0)|_{P}^{2}$, as to be proven.

From (20), if matrices $P$ and $Q$ satisfy (19), the guaranteed performance level for our scheme (in terms of size of the upper bound for index $J$ in (18) along solutions) is proportional to the inverse of $\eta \in(0,1)$. Then large values of $\eta<1$ lead to improved upper bounds of the LQ performance along solutions. On the other hand, from (8) and (9), smaller values of $\eta$ correspond to strictly smaller jump sets (and larger flow sets), which reveals that solutions are expected to flow longer before a switch of control input $\sigma$. Therefore one may use parameter $\eta$ to find a trade off between suitable transient switching frequency and transient performance along solutions.

In light of Theorem 2, we may tune the parameters $P, Q$ following an optimization capturing the goal of reducing as much as possible the right hand side of (20). To this end, we make the following natural selection:

$$
Q=C^{T} C+\nu I
$$

where $\nu>0$ is a (typically small) positive constant, which may be zero if $C^{T} C>0$.

Since $Q$ in (22) satisfies (19), under the assumption that the convex combination $\sum_{i=1}^{N} \lambda_{e, i} A_{i}$ is Hurwitz, the following convex optimization expressed by linear matrix inequalities always leads to a feasible solution:

$$
\begin{array}{ll}
\min _{P=P^{T}>0} & \operatorname{Trace}(P), \text { subject to: } \\
& \sum_{i=1}^{N} \lambda_{e, i} A_{i}^{T} P+P \sum_{i=1}^{N} \lambda_{e, i} A_{i}^{T} \leq-2 Q,
\end{array}
$$

and this optimized selection clearly satisfies Property 1, while minimizing the upper bound in (20).

\section{Practical global Results using SPACE- OR TIME-REGULARIZATION}

The hybrid control law proposed above can provide arbitrarily fast switching as the solution approaches $x_{e}$. In particular, given an initial condition in $\mathcal{A}$, one sees that the hybrid dynamics (6)-(9) has at least one solution that keeps jumping onto $\mathcal{A}$ without flowing. Infinitely fast switching is not desirable in terms of energy efficiency and reliability in many applications, such as power converters, because every switch dissipates energy and reduces the switch lifespan. For this reason, we propose a redesign of the hybrid law, aiming at reducing the number of switches when $\tilde{x}=x-x_{e}$ is close to zero, and avoiding infinitely fast switching. This goal is reasonable for the proposed law, because it is possible to show that away from $\mathcal{A}$, during transients, our control law already enjoys a desirable property of positive dwell time between switches, as long as Assumption 1 and Property 1 hold. To do so, we change system (6)-(9) with shorthand notation
$\mathcal{H}:=(f, G, \mathcal{C}, \mathcal{D})$, for a non-negative scalar $\varepsilon$, to the redesigned system:

$$
\begin{aligned}
\mathcal{H}_{\varepsilon} & :=\left(f, G, \mathcal{C}_{\varepsilon}, \mathcal{D}_{\varepsilon}\right) \\
\mathcal{C}_{\varepsilon} & :=\mathcal{C} \cup\left\{(x, \sigma): V\left(x-x_{e}\right) \leq \varepsilon\right\} \\
\mathcal{D}_{\varepsilon} & :=\mathcal{D} \cap\left\{(x, \sigma): V\left(x-x_{e}\right) \geq \varepsilon\right\},
\end{aligned}
$$

with $V$ as in (12). A useful practical dwell-time property for $\mathcal{H}$ is then established next. Lemma 2 below is a nontrivial consequence of the fact that Zeno solutions can only occur at the equilibrium $x_{e}$ for the hybrid closed loop. The ensuing dwell-time results are key to proving the properties of the regularized dynamics of this section.

Lemma 2: There exists a positive scalar $T^{*}$ such that for each $0<T \leq T^{*}$, there exists a scalar $\varepsilon>0$ such that all solutions to $\mathcal{H}$ jumping from set $\mathcal{D}_{\varepsilon}$ flow for at least $T$ ordinary time units after the jump, before reaching again set $\mathcal{D}_{\varepsilon}$. Moreover, as $T$ tends to zero, we have that $\varepsilon$ tends to zero as well.

Proof. To prove the lemma, it is enough to fix any scalar $\varepsilon=\varepsilon^{*}$ in (24) and show that there exists $T^{*}$ such that all solutions starting from $\mathcal{D}_{\varepsilon^{*}}$ flow for at least $T^{*}$ ordinary time units after the jump before reaching set $\mathcal{D}$. The rest of the lemma follows trivially from the fact that smaller values of $\varepsilon<\varepsilon^{*}$ are associated with the solutions starting in $\mathcal{D}_{\varepsilon^{*}}$ (already characterized by $T^{*}$ ) plus additional solutions starting in the compact set $\overline{\mathcal{D}_{\varepsilon} \backslash \mathcal{D}_{\varepsilon^{*}}}$, that enjoy a dwell-time property because any jump from this set maps to the interior of the flow set (and then one can consider the minimum flowing time over this compact set of initial conditions). Without loss of generality we can impose that the dwell time $T$ converge to zero as $\varepsilon$ converges to zero, thereby defining the function $\varepsilon$ discussed in the lemma.

Let us then fix a scalar $\varepsilon=\varepsilon^{*}$ in (24) and first notice that any solution jumping from $\mathcal{D}_{\varepsilon^{*}}$ at time $\left(t_{j}, j-1\right)$ satisfies, before and after the jump:

$$
\left|\tilde{x}\left(t_{j}, j\right)\right|_{Q}^{2}:=\tilde{x}^{T} Q \tilde{x} \geq q_{m}|\tilde{x}|^{2} \geq \frac{q_{m}}{p_{M}} V(\tilde{x}) \geq \frac{q_{m} \varepsilon^{*}}{p_{M}}=: 2 \varepsilon_{Q},
$$

where the dependence on $(t, j)$ has been omitted at the right-hand side, and where we denoted by $q_{m}$ and $q_{M}$ the minimum and maximum eigenvalues of $Q$, respectively, and by $p_{M}$ the maximum eigenvalue of $P$. Define now the function $\chi(\tau):=2 \varepsilon_{Q}-\left|\tilde{x}\left(t_{j}+\tau, j\right)\right|_{Q}^{2}$ and notice that (25) implies $\chi(0) \leq 0$. Consider now the flow dynamics in (7) and introduce scalars $b_{\sigma}=A_{\sigma} x_{e}+a_{\sigma}$ to get

$$
\dot{\tilde{x}}=A_{\sigma} x+a_{\sigma}=A_{\sigma} \tilde{x}+b_{\sigma},
$$

so that we may characterize the variation of $\chi$ as:

$$
\dot{\chi}=-2 \tilde{x}^{T} Q\left(A_{\sigma} \tilde{x}+b_{\sigma}\right) \leq \kappa_{1}|\tilde{x}|_{Q}^{2}+\kappa_{2}|\tilde{x}|_{Q},
$$

where $\kappa_{1}:=2 \frac{q_{M}}{q_{m}} \max _{\sigma \in \bar{N}}\left|A_{\sigma}\right|$ and $\kappa_{2}:=2 \frac{q_{M}}{\sqrt{q_{m}}} \max _{\sigma \in \bar{N}}\left|b_{\sigma}\right|$. Using now $|\tilde{x}|_{Q}^{2} \leq|\chi|+2 \varepsilon_{Q}$, which also gives $|\tilde{x}|_{Q} \leq \sqrt{|\chi|}+$ $\sqrt{2 \varepsilon_{Q}}$, because $|\chi|$ and $\varepsilon_{Q}$ are both non-negative, we get the bound:

$$
\begin{aligned}
\dot{\chi}(\tau) & \leq \kappa_{1}\left(|\chi(\tau)|+2 \varepsilon_{Q}\right)+\kappa_{2}\left(\sqrt{|\chi(\tau)|}+\sqrt{2 \varepsilon_{Q}}\right) \\
& =\kappa_{1}|\chi(\tau)|+\kappa_{2} \sqrt{|\chi(\tau)|}+\kappa_{3}, \quad \forall \tau \leq t_{j+1}-t_{j},
\end{aligned}
$$


where $\kappa_{3}=2 \kappa_{1} \varepsilon_{Q}+\kappa_{2} \sqrt{2 \varepsilon_{Q}}>0$. Denote by $\phi$ the solution to the differential equation induced by (28) starting at zero. This solution is continuous by definition, and strictly increasing because $\kappa_{i}>0$ for all $i=1,2,3$. Then there exists $T_{1}$ such that $\phi\left(T_{1}\right)=\varepsilon_{Q}$ and from standard comparison theory, and recalling that $\chi(0) \leq 0$ (by (25)), we have $\chi(\tau) \leq \varepsilon_{Q}$ for all $\tau \leq T_{1}$, which implies

$$
\left|\tilde{x}\left(t_{j}+\tau, j\right)\right|_{Q}^{2}=2 \varepsilon_{Q}-\chi(\tau) \geq \varepsilon_{Q}, \quad \forall \tau \leq T_{1} .
$$

Consider now equation (17) and define the function ${ }^{1}$

$$
\varsigma(\tilde{x}):=\frac{\tilde{x}^{T}\left(A_{\sigma} \tilde{x}+b_{\sigma}\right)}{|\tilde{x}|_{Q}^{2}}+1,
$$

which, from (17) clearly satisfies $\varsigma\left(\tilde{x}\left(t_{j}, j\right)\right) \leq 0$ after the jump from $\mathcal{D}_{\varepsilon^{*}}$. We prove below the existence of $T^{*}$ such that

$$
\varsigma\left(\tilde{x}\left(t_{j}+\tau, j\right)\right) \leq 1-\eta, \quad \text { for all } \tau \leq T^{*},
$$

which trivially proves $\tilde{x}\left(t_{j}+\tau, j\right)^{T}\left(A_{\sigma} \tilde{x}\left(t_{j}+\tau, j\right)+b_{\sigma}\right) \leq$ $-\eta\left|\tilde{x}\left(t_{j}+\tau, j\right)\right|_{Q}^{2}$ for all $\tau \leq T^{*}$, which in turn implies that the solution does not belong to $\mathcal{D}$, thus completing the proof of the lemma.

To prove (30), we proceed again with bounding the derivative of $\varsigma$. Straightforward derivations provide, along flowing solutions according to (7):

$$
\begin{aligned}
\dot{\varsigma} & =-\frac{2 \tilde{x}^{T} P\left(A_{\sigma} \tilde{x}+b_{\sigma}\right) \tilde{x}^{T} Q\left(A_{\sigma} \tilde{x}+b_{\sigma}\right)}{|\tilde{x}|_{Q}^{4}} \\
& +\frac{\tilde{x}^{T}\left(P A_{\sigma}+A_{\sigma}^{T} P\right) A_{\sigma} \tilde{x}+\tilde{x}^{T}\left(2 A_{\sigma}^{T} P+P A_{\sigma}\right) b_{\sigma}+b_{\sigma}^{T} P b_{\sigma}}{|\tilde{x}|_{Q}^{2}} \\
& \leq \varsigma_{1}+\varsigma_{2} \frac{1}{|\tilde{x}|_{Q}}+\varsigma_{3} \frac{1}{|\tilde{x}|_{Q}^{2}},
\end{aligned}
$$

where $\varsigma_{1}, \varsigma_{2}, \varsigma_{3}$ are sufficiently large positive scalars (and where we used $|\tilde{x}| \leq \frac{1}{\sqrt{q_{m}}}|\tilde{x}|_{Q}$ in several places). Consider now any time $\tau \leq T_{1}$, and use bound (29) to obtain $\dot{\varsigma} \leq \varsigma_{1}+\varsigma_{2} \varepsilon_{Q}^{-1 / 2}+\varsigma_{3} \varepsilon_{Q}^{-1}$, which, together with $\varsigma\left(\tilde{x}\left(t_{j}, j\right)\right) \leq 0$, and integrating $\dot{\varsigma}$, immediately gives $(30)$ for $T^{*}:=\min \left\{T_{1}, T_{2}\right\}$, where $T_{2}:=\frac{1-\eta}{\varsigma_{1}+\varsigma_{2} \varepsilon_{Q}^{-1 / 2}+\varsigma_{3} \varepsilon_{Q}^{-1}}$.

Lemma 2 ensures that a positive dwell time holds if solutions remain sufficiently far from $\mathcal{A}$. Then we have two possibilities to modify our control law to ensure that dwell time is enjoyed by solutions. One of them corresponds to replacing the jump set $\mathcal{D}$ by the restricted version in $\mathcal{D}_{\varepsilon}$ (we call it space regularization) and forcing solutions to flow in $\mathcal{D} \backslash \mathcal{D}_{\varepsilon}$ (this is called space regularization and is addressed in Section V-A), and the other one corresponds to forcing solutions not to jump unless some dwell time has expired (this is called time regularization and is addressed in Section V-B). Then, it makes sense to introduce the following $\varepsilon$-inflated version of attractor $\mathcal{A}$ :

$$
\mathcal{A}_{\varepsilon}:=\{(\tilde{x}, \sigma): V(\tilde{x}) \leq \varepsilon, \sigma \in \bar{N}\},
$$

\footnotetext{
${ }^{1}$ To avoid overloading notation, the hybrid time is only specified on the $\tilde{x}$ component, but the state variable $\sigma$ should be evaluated at the same hybrid time in the derivations at the end of the proof of Lemma 2.
}

which evidently reduces to $\mathcal{A}$ as $\varepsilon$ tends to zero. Practical stabilization of $\mathcal{A}$ comprises finding a parametric control law (whose parameter is $\varepsilon$ ) such that for each sufficiently small value of $\varepsilon$ a subset of $\mathcal{A}_{\varepsilon}$ is UGAS for the closed loop. This is done in the next sections.

\section{A. Space regularization}

Based on Lemma 2, for any value of a positive scalar $\varepsilon$, let us consider the space-regularized version of $\mathcal{H}=$ $(f, G, \mathcal{C}, \mathcal{D})$ given in $(24)$. The regularized dynamics are clearly motivated by the fact that jumps are forbidden when solutions are $\varepsilon$-close to the attractor.

Mainly using Lemma 2 the following desirable results are enjoyed by hybrid system $\mathcal{H}_{\varepsilon}$.

Theorem 3: Consider point $x_{e}$ and a vector $\lambda_{e}$ satisfying Assumption 1 and matrices $P \in \mathbb{R}^{n \times n}$ and $Q \in \mathbb{R}^{n \times n}$ satisfying Property 1 . The following hold:

1) for any positive scalar $\varepsilon$, set $\mathcal{A}_{\varepsilon}$ in (31) is UGAS for dynamics $\mathcal{H}_{\varepsilon}$ in $(24)$;

2 ) set $\mathcal{A}$ is globally practically asymptotically stable for (24), with respect to parameter $\varepsilon$;

3) There exists $T>0$ such that all solutions to $\mathcal{H}_{\varepsilon}$ enjoy a $T$-dwell-time property, namely given any solution $\varphi$ to $\mathcal{H}_{\varepsilon}$, all $(t, j) \in \operatorname{dom} \varphi$ satisfy $t \geq \frac{j}{T}-1$.

Proof. First notice that sets $\mathcal{C}_{\varepsilon}$ and $\mathcal{D}_{\varepsilon}$ are both closed. Indeed, $\mathcal{C}_{\varepsilon}$ is the union of two closed sets and $\mathcal{D}_{\varepsilon}$ is the intersection of two closed sets. Then, due to the properties of $f$ and $G$, system $\mathcal{H}_{\varepsilon}$ satisfies the hybrid basic conditions of [7, As. 6.5] and we may apply several useful results pertaining to well-posed hybrid systems.

Proof of item 3). This item follows in a straightforward way from Lemma 2 . Indeed, solutions to $\mathcal{H}_{\varepsilon}$ can only jump from $\mathcal{D}_{\varepsilon}$. Any such solution $\varphi$ flows for at least $T$ time after each jump, before reaching again $\mathcal{D}_{\varepsilon}$, which clearly implies $t+1 \geq \frac{j}{T}$ (where the "1" takes care of the initial condition), as to be proven.

Proof of item 1). Consider the following Lyapunov function candidate:

$$
V_{\varepsilon}(\tilde{x})=\max \{V(\tilde{x})-\varepsilon, 0\},
$$

which is clearly positive definite with respect to $\mathcal{A}_{\varepsilon}$ and radially unbounded. Since outside set $\mathcal{A}_{\varepsilon}$ the hybrid dynamics $\mathcal{H}_{\varepsilon}$ coincides with the one of $\mathcal{H}$, then equations (13) and (14) hold for any $(\tilde{x}, \sigma)$ not in $\mathcal{A}_{\varepsilon}$, which implies that

$$
\begin{array}{lc}
\left\langle\nabla V_{\varepsilon}(\tilde{x}), f(x, \sigma)\right\rangle<0 & \forall \tilde{x} \in \mathcal{C}_{\varepsilon} \backslash \mathcal{A}_{\varepsilon} \\
V_{\varepsilon}\left(\tilde{x}^{+}\right)-V_{\varepsilon}(\tilde{x})=0, & \forall \tilde{x} \in \mathcal{D}_{\varepsilon} \backslash \mathcal{A}_{\varepsilon}
\end{array}
$$

Moreover, from the property established in item 3), all complete solutions to $\mathcal{H}_{\varepsilon}$ must flow for some time, and therefore from (33), we have that no solution can keep $V_{\varepsilon}$ constant and non-zero. UGAS of $\mathcal{A}_{\varepsilon}$ by applying the nonsmooth invariance principle in [19], also using the well posedness result established at the beginning of the proof.

Proof of item 2). The proof follows in a straightforward way from the previous item, after noticing that given any neighborhood $\mathcal{I}$ of $\mathcal{A}$, there exists a small enough $\varepsilon>0$ such that $\mathcal{A}_{\varepsilon} \subset \mathcal{I}$. 


\section{B. Time regularization}

Based on Lemma 2, for any value of $T<T^{*}$, we may introduce the following additional state variable $\tau$ to dynamics (6):

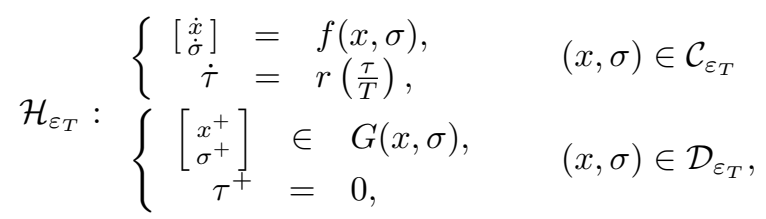

where $r(s):=\min \{1,2-s\}$, for all $s \geq 0$ and the jump and flow sets are the following time-regularized versions of $\mathcal{C}$ and $\mathcal{D}$ in $(6)-(9)$ :

$$
\begin{aligned}
& \mathcal{C}_{\varepsilon_{T}}:=\mathcal{C} \times[0,2 T] \cup\{(x, u, \tau): \tau \in[0, T]\} \\
& \mathcal{D}_{\varepsilon_{T}}:=\mathcal{D} \times[T, 2 T] .
\end{aligned}
$$

The above regularization is clearly motivated by the fact that jumps are forbidden when the timer $\tau$ is too small, namely not enough time has elapsed since the last jump. Then all solutions are forced to flow for at least $T$ ordinary time after each jump. Note also that function $r$ at the right-hand side of equation (35a) allows a solution to flow forever while ensuring that timer $\tau$ remains in a compact set.

Before proceeding any further, we emphasize that forcing a solution to flow regardless of whether it belongs to $\mathcal{D}$ or not, may lead to an increase of function $V$. It is useful to quantify how much increase $V$ can experience from the set where $V(\tilde{x}) \leq \varepsilon_{T}$ (let recall $\varepsilon_{T}:=\varepsilon$, being $\varepsilon$ introduced in Lemma 2). To this end, we exploit the affine nature of the dynamics and observe that along solutions of (35) we have $\dot{V}(\tilde{x}) \leq|\tilde{x}||P||\dot{\tilde{x}}|=|\tilde{x}||P||\dot{\tilde{x}}| \leq$ $|\tilde{x}||P|\left(\kappa_{1}|\tilde{x}|+\kappa_{2}\right) \leq 2 \alpha V(\tilde{x})+2 \beta \sqrt{V(\tilde{x})}$, where $\alpha$ and $\beta$ are large enough positive scalars and where we used positive definiteness of $P$ and the sector growth condition $|\dot{\tilde{x}}|=|\dot{x}| \leq\left|A_{\sigma}\left(x-x_{e}\right)\right|+\left|A_{\sigma} x_{e}+a_{\sigma}\right| \leq \kappa_{1}|\tilde{x}|+\kappa_{2}$ (which clearly holds for some $\kappa_{1}>0$ and $\kappa_{2}>0$ ). Proceeding as in [11, page 203], we obtain along any solution $\phi$ satisfying $(t, j) \in \operatorname{dom} \phi$ and $(t+T, j) \in \operatorname{dom} \phi$,

$$
\begin{aligned}
& \sqrt{V(\phi(t+\tau, j))} \leq \mathrm{e}^{\alpha \tau} \sqrt{V(\phi(t, j))}+\beta \int_{0}^{\tau} \mathrm{e}^{\alpha s} d s \\
&=\mathrm{e}^{\alpha \tau} \sqrt{V(\phi(t, j))}+\frac{\beta}{\alpha}\left(\mathrm{e}^{\alpha \tau}-1\right), \quad \forall \tau \in[0, T] .
\end{aligned}
$$

Therefore, assuming that $V(\phi(t, j)) \leq \varepsilon_{T}$, we obtain for all $\tau \in[0, T]$,

$$
V(\phi(t+\tau, j)) \leq \varepsilon_{T}(T):=2 \mathrm{e}^{2 \alpha T} \varepsilon_{T}+\frac{2 \beta^{2}}{\alpha^{2}}\left(\mathrm{e}^{\alpha T}-1\right)^{2} .
$$

This bound motivates introducing the following set:

$$
\mathcal{E}_{T}:=\left\{(\tilde{x}, \sigma, \tau): V(\tilde{x}) \leq \varepsilon_{T}(T), \sigma \in \bar{N}, \tau \in[0,2 T]\right\},
$$

which enjoys the nice property of shrinking to $\mathcal{A}_{\varepsilon} \times\{0\}$, as $T$ converges to zero.

Mainly using Lemma 2 the following desirable results are enjoyed by hybrid system $\mathcal{H}_{\varepsilon_{T}}$ in (35).
Theorem 4: Consider point $x_{e}$ and a vector $\lambda_{e}$ satisfying Assumption 1 and matrices $P \in \mathbb{R}^{n \times n}$ and $Q \in \mathbb{R}^{n \times n}$ satisfying Property 1 . The following holds:

1) all solutions to $\mathcal{H}_{\varepsilon_{T}}$ enjoy a dwell-time property corresponding to $T$;

2) for any positive scalar $T<T^{*}$, there exists a compact set $\mathcal{A}_{\varepsilon} \times[0,2 T] \subset \mathcal{E}_{T}$, which is UGAS for dynamics $\mathcal{H}_{\varepsilon_{T}}$ in (35);

3) set $\mathcal{A} \times\{0\}$ is globally practically asymptotically stable for (35), with respect to parameter $T$ (namely as long as $T$ is sufficiently small, the UGAS set $\mathcal{A}_{\varepsilon} \times[0,2 T]$ characterized in the previous item can be made arbitrarily close to $\mathcal{A} \times\{0\})$.

Proof. Similar to the proof of Theorem 3 we start by noticing that hybrid system (35) enjoys the hybrid basic conditions of $\left[7\right.$, As. 6.5], because sets $\mathcal{C}_{\varepsilon_{T}}$ and $\mathcal{D}_{\varepsilon_{T}}$ are both closed and $f$ and $G$ enjoy desirable properties. Then we may apply several useful results pertaining well-posed hybrid systems (specifically, in the proof of item 2 below).

Proof of item 1. The dwell-time property of solutions follows in a straightforward way from the fact that solutions are forced to not jump until the timer variable $\tau$ has reached the value $T$. Since $\dot{\tau}=1$ for all $\tau \leq T$, then all solutions flow for at least $T$ ordinary time after each jump (because $\tau^{+}=0$ across jumps).

Proof of item 2. Consider the two hybrid systems $\mathcal{H}_{\varepsilon}$ and $\mathcal{H}_{\varepsilon_{T}}$ in (24) and (35), respectively. For any positive value of $T<T^{*}$, we have shown in the proof of item 1 of Theorem 3 that it suffices to pick $\varepsilon_{T}=\varepsilon$ (coming $\varepsilon$ from Lemma 2) to obtain UGAS of the attractor $\mathcal{A}_{\varepsilon}$ in (31) and a dwell time of $T$ for all solutions to $\mathcal{H}_{\varepsilon}$. Since the $(x, \sigma)$ dynamics of $\mathcal{H}_{\varepsilon}$ and $\mathcal{H}_{\varepsilon_{T}}$ coincide, except for the dwell-time restriction on $\mathcal{H}_{\varepsilon_{T}}$, the above mentioned dwelltime property of solutions to $\mathcal{H}_{\varepsilon}$ ensures that (possibly after an initial flow of at most $T$ ordinary time) the $(x, \sigma)$ component of each solution to $\mathcal{H}_{\varepsilon_{T}}$ remaining outside $\mathcal{A}_{\varepsilon} \times$ $[0,2 T]$, coincides with a solution to $\mathcal{H}_{\varepsilon}$, therefore any such solution to $\mathcal{H}_{\varepsilon_{T}}$ must approach $\mathcal{A}_{\varepsilon} \times[0,2 T]$, which is a strict subset of $\mathcal{E}_{T}$ in (37). Two things may happen then. Either the solution approaches $\mathcal{A}_{\varepsilon} \times[0,2 T]$ without ever reaching it, so it eventually remains in $\mathcal{E}_{T}$, or it reaches $\mathcal{A}_{\varepsilon} \times[0,2 T]$ and may then be forced to flow by the dwelltime logic of $\mathcal{H}_{\varepsilon_{T}}$. However, in this last case we get from bound (36) that such a solution cannot flow outside $\mathcal{E}_{T}$. As a consequence, $\mathcal{E}_{T}$ is uniformly attractive and reached in finite time by all solutions, in addition to being strongly forward invariant for $\mathcal{H}_{\varepsilon_{T}}$.

We now use the well-posedness property established at the beginning of the proof to exploit a number of regularity results from [7, Ch. $6 \&$ 7]. Denote by $\Omega\left(\mathcal{E}_{T}\right)$ the $\omega$-limit set of $\mathcal{E}_{T}$ (see [7, Def. 6.23]) and note that it cannot be empty, and must satisfy $\Omega\left(\mathcal{E}_{T}\right) \subset \mathcal{E}_{T}$, because $\mathcal{E}_{T}$ is bounded and strongly forward invariant. Then using again strong forward invariance of $\mathcal{E}_{T}$ we get boundedness of all solutions starting from $\mathcal{E}_{T}$ and we may apply [7, Prop. 6.26] to obtain that $\Omega\left(\mathcal{E}_{T}\right)$ is compact, nonempty, uniformly attractive from $\mathcal{E}_{T}$, and strongly forward invariant. Since also $\mathcal{E}_{T}$ is uniformly attractive, we may then apply a global 
version ${ }^{2}$ of [7, Prop. 7.5] applied to the compact attractor $\Omega\left(\mathcal{E}_{T}\right)$, to conclude global asymptotic stability of $\Omega\left(\mathcal{E}_{T}\right)$, which is equivalent to UGAS from [7, Th $3.40 \&$ Th 7.12].

Proof of item 3. Item 3 follows in a straightforward way by recalling from Lemma 2 that $\varepsilon$ converges to zero as $T$ goes to zero, and then that also $\varepsilon(T)$ in (36) enjoys the same property. As a consequence, set $\mathcal{E}_{T}$ in (37) shrinks to $\mathcal{A} \times\{0\}$ as $T$ goes to zero, and since we established in item 2 that $\mathcal{A}_{\varepsilon} \times[0,2 T] \subset \mathcal{E}_{T}$ for all $T>0$, we can make $\mathcal{A}_{\varepsilon} \times[0,2 T]$ arbitrarily close to $\mathcal{A} \times\{0\}$ by selecting $T$ sufficiently small.

\section{Illustrative EXAmPle}

The two hybrid control schemes developed in Section V-A (space regularization) and V-B (time regularization) are tested on a boost converter model taken from [3]. The state variable is $x=\left[\begin{array}{ll}i_{L} & v_{C}\end{array}\right]^{T}$, where $i_{L}$ denotes the inductor current and, $v_{C}$ denotes the capacitor voltage. The switched system (1) is then defined by the matrices

$$
\begin{aligned}
& A_{1}=\left[\begin{array}{cc}
-\frac{R}{L} & 0 \\
0 & -\frac{1}{R_{0} C_{0}}
\end{array}\right], A_{2}=\left[\begin{array}{cc}
-\frac{R}{L} & -\frac{1}{L} \\
\frac{1}{C_{0}} & -\frac{1}{R_{0} C_{0}}
\end{array}\right], \\
& a_{1}=a_{2}=\left[\begin{array}{ll}
\frac{V_{i n}}{L} & 0
\end{array}\right]^{T},
\end{aligned}
$$

where the considered nominal values are: $V_{i n}=100 \mathrm{~V}$, $R=2 \Omega, L=500 \mu H, C_{o}=470 \mu F$ and $R_{o}=50 \Omega$. The desired equilibrium point is chosen as $x_{e}=\left[\begin{array}{ll}3 & 120\end{array}\right]^{T}$. Assumption 1 is therefore satisfied with $\lambda_{e}=\left[\begin{array}{ll}0.22 & 0.78\end{array}\right]$. We select the quadratic cost function in (18) as

$J=\sum_{k \in \operatorname{dom}_{j}(\xi)} \int_{t_{k}}^{t_{k+1}} \frac{\rho}{R_{0}}\left(v_{c}(\tau, k)-v_{e}\right)^{2}+R\left(i_{L}(\tau, k)-i_{e}\right)^{2} d \tau$,

where $\rho=1000$ to suitably penalize the voltage error. Multiplying the cost by $(\rho R)^{-1}$, one clearly sees that

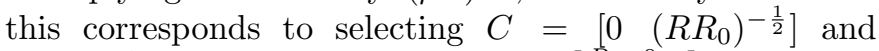
$\nu=\rho^{-1}$ in (22), which gives $Q=\left[\begin{array}{cc}R & 0 \\ 0 & \rho / R_{0}\end{array}\right]$. With this value of $Q$, in order to satisfy Property 1 , we choose $P=\left[\begin{array}{ll}0.5245 & 0.1032 \\ 0.1032 & 1.0596\end{array}\right]$.

In Figure 1 we report on the results of extensive simulation tests, and the arising statistics about the switching frequency. To suitably illustrate the different roles of the "transient" parameter $\eta$ introduced in Section III and the "steady-state" parameters $\varepsilon$ and $T$ introduced in Sections V-A and V-B, respectively, we select a grid of possible values of $(\eta, \varepsilon)$ (for the space regularization case, shown in the two upper surfaces), and a grid of possible values of $(\eta, T)$ (for the time regularization case, shown in the two bottom surfaces). Two large sets of simulations have been carried out using space regularization and time regularization, respectively, leading to Figure 1.

Let us first consider the upper surfaces of Figure 1 (space regularization). Each point on these surfaces correspond to a pair $(\eta, \varepsilon)$ and has been generated by first running eight simulations from eight different initial conditions, with

\footnotetext{
${ }^{2}$ A global version of [7, Prop. 7.5] is trivially obtained by establishing its hypotheses for any arbitrary positive value of $\mu$.
}
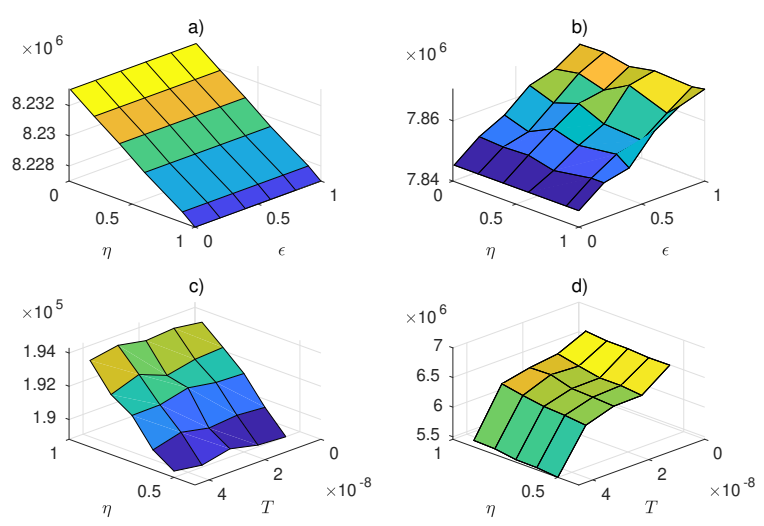

Fig. 1. Top surfaces: evolution of the switching frequency with space regularization in the transient (left) and at the steady state (right). Bottom surfaces: evolution of the average switching frequency with time regularization in the transient (left) and at the steady state (right).
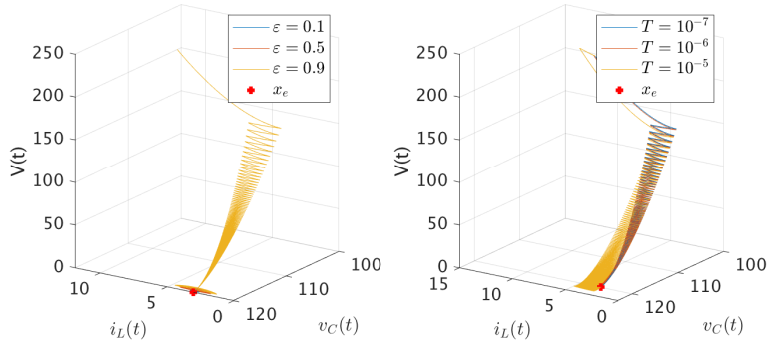

Fig. 2. Lyapunov function $V(\tilde{x})$ with space regularization (left) and with time regularization (right).

good coverage of all the possible directions of the initial error. These initial conditions all correspond to an initial value of $V(\tilde{x}(0,0))=200$ (they are all on the same level set of $V$ ). Each simulation runs for $50 \mathrm{~ms}$ and the statistics reported in the left of Figure 1 show the average swtiching frequency in the time domain preceding the first time $(\bar{t}, \bar{j}) \in \operatorname{dom} \tilde{x}$ when $V(\tilde{x}(\bar{t}, \bar{j})) \leq \varepsilon$ (when the response is still in the transient phase). The right surface shows instead the response in the remaining portion of each simulation (where the response has reached the steady state). Each of these statistics represents the number of switches normalized by the length of the interval, averaged over the eight simulated solutions. We may appreciate the fact that the steady-state parameter $\varepsilon$ has no effect on the transient switching frequency and has significant effect on the steady-state switching frequency. The converse holds for the transient parameter $\eta$, which is shown to have an effect on the transient switching frequency.

Time regularization is instead used in the lower surfaces of Figure 1, corresponding to a grid of selections of the two parameters $(\eta, T)$, where for each point on the grid eight simulations from the same initial conditions as in the previous case, are performed. For this second case, a rough indication of the expiration of the transient phase has been performed by detecting the smallest time $(\bar{t}, \bar{j})$ when $V(\tilde{x}(\bar{t}, \bar{j})) \leq 1$ namely it is 200 times smaller than 
the initial condition), and transient statistics (providing the lower left surface of Figure 1) is the averaged switching frequency over hybrid times up to $t=\bar{t} / 2$, whereas the steady-state statistics (providing the lower right surface of Figure 1) are computed by focusing on hybrid times after $\frac{3}{2} \bar{t}$ and until the end of the simulation run. The resulting two lower plots of Figure 1 confirm the same trends as in the space regularization case, even though here the steadystate tuning knob is given by scalar $T$.

Figure 2 shows the evolution of the Lyapunov function (12) with space regularization (left) and time regularization (right) for the same initial condition and different selections of the steady-state parameters $\varepsilon$ and $T$. In the left plot, we may see that as $\varepsilon$ is decreased, the solution comes closer to the operating point $x_{e}$ but, as noticed in Section III, the price to pay for such proximity is a high average switching frequency (indeed, $x_{e}$ is not an equilibrium for the two dynamics of the switching scheme). Conversely, for larger values of $\varepsilon$, the number of jumps decreases and, as expected, the error between $x$ and $x_{e}$ increases. Similarly, for the right plot of Figure 2, smaller values of $T$ provide solutions that remain increasingly close to $x_{e}$ exhibiting a large switching frequency, and viceversa.

\section{CONClusions AND FUture WORK}

We addressed practical stabilization of operating points for switched affine systems by using a hybrid controller that performs a trade off between minimum dwell time and the size of the asymptotically stable set. Practical asymptotic stability is obtained by two design strategies, involving space- and time-regularization. Each one of these strategies is associated to a convenient tuning knob that may be used to perform a trade-off between the dwell time and the magnitude of the steady-state oscillations around the operating point. The switching frequency during the transient phase of the response can also be adjusted using another convenient knob, having the intuitive role of trading off transient dwell time with LQ guarantees, as seen from an integral quadratic performance index. The proposed construction has been numerically illustrated on a boost converter example. Future work comprises experimental validation of the proposed strategy, as well as possible generalizations requiring weaker conditions on the system matrices $A_{i}, i=1,2, \ldots, n$.

\section{ACKNOWLEDGMENTS}

This work has been partially funded under grant "HISPALIS" ANR-18-CE40-0022-01.

\section{REFERENCES}

[1] C. Albea, G. Garcia, and L. Zaccarian. Hybrid dynamic modeling and control of switched affine systems: application to DC-DC converters. In IEEE Conference on Decision and Control, pages 2264-2269, Osaka, Japan, 2015.

[2] J. Buisson, P.Y. Richard, and H. Cormerais. On the stabilisation of switching electrical power converters. In Hybrid Systems: Computation and Control, volume 3414 of Lecture Notes in Computer Science, pages 184-197. Springer Berlin Heidelberg, 2005.
[3] G. S. Deaecto, J. C. Geromel, F.S. Garcia, and J.A. Pomilio. Switched affine systems control design with application to DC-

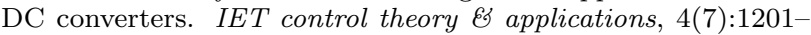
$1210,2010$.

[4] L. Dieci and N. Guglielmi. Regularizing piecewise smooth differential systems: co-dimension 2 discontinuity surface. Journal of Dynamics and Differential Equations, 25(1):71-94, 2013.

[5] E. Feron. Quadratic stabilizability of switched systems via state and output feedback. Report, Center for Intelligent Control Systems, Laboratory for Information and Decision Systems, MIT, 1996.

[6] J.C. Geromel and P. Colaneri. Stability and stabilization of continuous-time switched linear systems. SIAM Journal on Control and Optimization, 45(5):1915-1930, 2006.

[7] R. Goebel, R.G. Sanfelice, and A.R. Teel. Hybrid Dynamical Systems: modeling, stability, and robustness. Princeton University Press, 2012.

[8] P. Hauroigne, P. Riedinger, and C. Iung. Switched affine systems using sampled-data controllers: Robust and guaranteed stabilisation. IEEE Trans. on Automatic Control, 56(12):29292935, 2011.

[9] L. Hetel and E. Bernuau. Local stabilization of switched affine systems. IEEE Transactions on Automatic Control, 60(4):11581163, 2015.

[10] L. Hetel and E. Fridman. Robust sampled-data control of switched affine systems. IEEE Trans. on Automatic Control, 58(11):2922-2928, 2013.

[11] H.K. Khalil. Nonlinear systems, vol. 3. Prentice Hall, 2002.

[12] D. Liberzon. Switching in systems and control. Birkhauser, 2003.

[13] D. Liberzon and A.S. Morse. Basic problems in stability and design of switched systems. IEEE Control Systems Magazine, 19(5):59-70, 1999.

[14] J. Liu, X. Liu, and W.C. Xie. On the $\left(h_{0}, h\right)$-stabilization of switched nonlinear systems via state-dependent switching rule. Applied Mathematics and Computation, 217(5):2067-2083, 2010.

[15] Y. Lu and W. Zhang. A piecewise smooth control-lyapunov function framework for switching stabilization. Automatica, 76:258-265, 2017.

[16] C. Prieur, A.R. Teel, and L. Zaccarian. Relaxed persistent flow/jump conditions for uniform global asymptotic stability. IEEE Trans. on Automatic Control, 59(10):2766-2771, 2014.

[17] C. Seatzu, D. Corona, A. Giua, and A. Bemporad. Optimal control of continuous-time switched affine systems. IEEE Transactions on Automatic Control, 51(5):726-741, 2006.

[18] M. Senesky, G. Eirea, and T.J. Koo. Hybrid modelling and control of power electronics. In Hybrid Systems: Computation and Control, volume 2623 of Lecture Notes in Computer Science, pages 450-465. Springer Berlin Heidelberg, 2003.

[19] A. Seuret, C. Prieur, S. Tarbouriech, A.R. Teel, and L. Zaccarian. A nonsmooth hybrid invariance principle applied to robust event-triggered design. Submitted to IEEE Transactions on Automatic Control. See also: https://hal.archivesouvertes.fr/hal-01526331/, 2017.

[20] E. Skafidas, R. J Evans, A.V. Savkin, and I.R. Petersen. Stability results for switched controller systems. Automatica, 35(4):553-564, 1999.

[21] Z. Sun. A robust stabilizing law for switched linear systems. International Journal of Control, 77(4):389-398, 2004.

[22] T. A.F. Theunisse, J. Chai, R.G. Sanfelice, and W.P. Heemels. Robust global stabilization of the DC-DC boost converter via hybrid control. IEEE Trans. on Circuits and Systems I: Regular Papers, 62(4):1052-1061, 2015.

[23] A.J. Van Der Schaft and J.M. Schumacher. An introduction to hybrid dynamical systems, volume 251. Springer London, 2000.

[24] L. Zhang and P. Shi. Stability, $l_{2}$-gain and asynchronous $H_{\infty}$ control of discrete-time switched systems with average dwell time. IEEE Trans. on Automatic Control, 54(9):2192-2199, 2009 . 